\title{
FEATURE FUSION WITH NEIGHBORHOOD- OSCILLATING TABU SEARCH FOR ORIENTED TEXTURE CLASSIFICATION
}

Yindi Zhao, Liangpei Zhang, and Pingxiang Li

LIESMARS, Wuhan Univiersity, Wuhan 430079, China

Abstract: $\quad$ This paper develops two techniques of oriented texture analysis: the modified Gabor filters (MGF) and the Gaussian Markov random field model with circular neighborhoods (CGMRF). The neighborhood-oscillating tabu search algorithm (NOTS) is proposed to solve the MGF/CGMRF feature fusion problem, and compared with classical algorithms, such as sequential forward selection and sequential forward floating selection methods. Based on the experimental results, NOTS is shown to be a promising tool for feature fusion, and the MGF/CGMRF fused features achieved by NOTS perform better than either MGF or CGMRF alone according to the Fisher criterion and classification accuracy.

Key words: Gabor filters, Gaussian Markov random field, tabu search, feature fusion

\section{INTRODUCTION}

With the increased demand for automatic inspection of industrial application, oriented texture analysis has received great attention in machine vision. Oriented textures are characterized by a dominant orientation at each point of the texture, and can be summarized by images encoding these dominant orientations. Many approaches of extracting texture features have been proposed over recent years, including spatial frequency based techniques such as multi-channel Gabor filters [1], stochastic models such as Markov random fields (MRF) [2] , and statistical analysis methods. Gabor filters can obtain multi-scale texture information corresponding to different scales and orientations. And MRF can capture the local spatial texture 
information assuming that the intensity in an image depends on the intensities of only the neighboring pixels. Based on traditional techniques, this paper develops two schemes of oriented texture analysis: the modified Gabor filters (MGF) and the Gaussian Markov random field model with circular neighborhoods (CGMRF).

MGF and CGMRF features extracted from an input oriented texture image are quite different in nature and have low inter-feature correlations [3]. The combined MGF and CGMRF features are expected to provide richer texture information than either MGF or CGMRF features alone. However, the combined features without selection give more dimensions, which may affect the performance of designed classifiers and result in even worse classification accuracy than the pure features. In order to reduce data quantity and improve data quality, the combination of different features should be processed by feature selection. In this paper, the neighborhoodoscillating tabu search algorithm (NOTS) is proposed to select an optimal feature subset from the pooled set of MGF and CGMRF features. The performance of NOTS is compared with some classical algorithms, such as sequential forward selection and sequential forward floating selection methods.

The rest of this paper is organized as follows. In the following section, MGF and CGMRF texture features are presented. In Section 3, NOTS is proposed to solve the MGF/CGMRF feature fusion problem. Experimental results are given in Section 4 and conclusions drawn in Section 5.

\section{ORIENTED TEXTURE FEATURE EXTRACTION}

\subsection{Multi-Channel Gabor Filters}

Each texture can be thought of as containing a narrow range of frequency and orientation components. By filtering an input texture image with multiple band-pass filters tuned to the dominant frequency and orientation component of the textures, it is possible to locate each texture. Due to its appealing simplicity and optimum joint spatial/spatial-frequency localization, the Gabor filter is attractive for texture analysis. The Gabor function takes the form of a 2-D Gaussian modulated complex sinusoidal grating in the spatial domain [1]: 


$$
h(x, y)=\frac{1}{2 \pi \sigma_{1} \sigma_{2}} \cdot \exp \left\{-\frac{1}{2}\left[\left(\frac{x^{\prime}}{\sigma_{1}}\right)^{2}+\left(\frac{y^{\prime}}{\sigma_{2}}\right)^{2}\right]\right\} \cdot \exp [2 \pi j(U x+V y)]
$$

where $\sigma_{1}$ and $\sigma_{2}$ characterize the spread in the $x$ and $y$ directions, $\left(x^{\prime}, y^{\prime}\right)=(x \cos \theta+y \sin \theta,-x \sin \theta+y \cos \theta)$ represents rotated spatialdomain rectilinear coordinates, $(U, V)$ defines the position of the filter in the frequency domain with a center frequency of $F=\sqrt{U \times U+V \times V}$ and an orientation of $\phi=\arctan (V / U)$.

In order to maximize the coverage of the frequency domain while minimizing the overlap between filters, there are another two important aspects, namely the frequency bandwidth $B_{F}$ (measured in octave) and the angular bandwidth $B_{\theta}$ (measured in radian). For more details on the frequency and angular bandwidth, the reader is encouraged to refer to [1, 4]. The frequency and angular bandwidth can be set to constant values that match psychovisual data. Jain and Farrokhnia [1] implemented Gabor filters for texture segmentation using frequency bandwidth $\left(B_{F}\right)$ of one octave, center frequency spacing $\left(S_{F}\right)$ of one octave, angular bandwidth $\left(B_{\theta}\right)$ of $45^{\circ}$ and angular spacing $\left(S_{\theta}\right)$ of $45^{\circ}$. For a $N \times N$ texture image, the center frequencies $F$ (normalized by $N$ ) are selected as follows:

$$
F=\sqrt{2}\{1,2, \quad 4, \quad 8, \cdots, \quad N / 4\} / N \text { cycles per pixel (cpp) }
$$

When the image width and height are not equal, in order to guarantee that the passband of the filter with the highest center frequency falls inside the image, the values of $F$ should be set based on the smaller side. On the other hand, due to the symmetry of Fourier spectrum, only half part $\left[0^{\circ}, 180^{\circ}\right]$ is considered. Thus 4 equally spaced orientations are used: $0^{\circ}, 45^{\circ}, 90^{\circ}$, and $135^{\circ}$. For the sake of simplicity, we call the above designed Gabor filters as traditional Gabor filters (TGF).

Unit octave spacing matches the experimentally determined human visual system ability; however, the $45^{\circ}$ angular bandwidth is not in agreement, and the $30^{\circ}$ angular bandwidth is argued to have stronger capability of capturing texture features [4]. In this paper, a smaller bandwidth of $22.5^{\circ}$ is used in order to detect oriented textures effectively. Finer quantization of orientation may be needed in general. And a bigger one will bring about negative impacts on the discrimination performance for oriented textures when there are texture orientations to which none of the channels of the filter bank will respond sufficiently. Furthermore, for an input oriented texture image of size 
$M \times N(M \neq N)$, the center frequencies determined by Equation 2 cannot cover the entire frequency domain because of frequency leakage. From this point we propose a new frequency selection scheme. First radial directions are defined corresponding to the angular bandwidth and angular spacing. Then draw a radial line from the image center along each radial direction, and compute the distance $(L)$ between the image center and the point of intersection of the radial line with the image side. For each direction, the center frequencies $F$ are set by the similar way of Equation 2:

$$
F=\sqrt{2}\{1, \quad 2, \quad 4, \quad 8, \cdots, \quad L / 2\} / L \mathrm{cpp}
$$

We denote the Gabor filters with 8 equidistant preferred orientations $\left(0^{\circ}\right.$, $22.5^{\circ}, 45^{\circ}, 67.5^{\circ}, 90^{\circ}, 112.5^{\circ}, 135^{\circ}$, and $\left.157.5^{\circ}\right)$ and center frequencies selected using the proposed new scheme as modified Gabor filters (MGF). However, Filter outputs by default are not appropriate for identifying key texture features. Each filtered image is subjected to a sigmoidal non linear transformation that is interpreted as a blob detector [1].

\subsection{MRF Model}

MRF can specify the local dependence of image regions by defining a neighborhood system on the pixels of an image and a probability density function on the spectrum distribution of the pixels. A typical MRF model is the Gaussian MRF (GMRF) model, which is widely used for modeling image textures. Let $i(s)$ represent the gray level intensity of a pixel $s$, the GMRF model is defined by the following equation:

$$
i(s)-u=\sum_{r \in \eta} \theta(r) \times(i(s+r)-u)+e(s)
$$

where $u$ is the mean of variable $i(s), \theta(r) \mathrm{s}$ are the model parameters and $\eta$ is a neighbor set, and $e(s)$ is a zero-mean Gaussian noise sequence with the variance of $v$.Since GMRF models are defined only for symmetric neighborhood sites, often $\eta$ is equivalently characterized using an asymmetrical neighbor set $\bar{\eta}$, i.e. if $r \in \bar{\eta}$ then $-r \notin \bar{\eta}$ and $\eta=\{r: r \in \bar{\eta}\} \cup\{-r: r \in \bar{\eta}\}$. Therefore, Equation 4 can be rewritten by

$$
i(s)-u=\sum_{r \in \bar{\eta}} \theta(r)((i(s+r)-u)+(i(s-r)-u))+e(s)
$$


There are many existing methods for estimating the GMRF model parameters. The choice of the least squares method [5] is motivated by this simplicity-stability tradeoff. Then the least square estimates of the parameters are:

$$
\hat{\boldsymbol{\theta}}=\left[\sum_{s \in R} \mathbf{q}(s) \mathbf{q}^{\mathrm{T}}(s)\right]^{-1}\left[\sum_{s \in R} \mathbf{q}(s)(i(s)-u)\right]
$$

where $\mathbf{q}(s)=\operatorname{col}[(i(s+r)-u)+(i(s-r)-u) \mid r \in \bar{\eta}], R$ is an interior of the image, $\hat{\boldsymbol{\theta}}=\operatorname{col}[\hat{\theta}(r) \mid r \in \bar{\eta}]$, and the hat $\wedge$ over a quantity indicates an estimate of that quantity. The estimate $\hat{v}$ of the noise variance is

$$
\hat{v}=\frac{1}{M} \sum_{s \in R}\left[(i(s)-u)-\hat{\boldsymbol{\theta}}^{\mathrm{T}} \mathbf{q}(s)\right]^{2}
$$

where $M$ demotes the number of elements in $R$. According to the GMRF model parameters $\hat{\theta}(r) \mathrm{s}$, a new different set of texture features, $f(r) \mathrm{s}$ are derived by [2]

$$
f(r)=\frac{1}{M} \sum_{s \in R}[(i(s)-u)-\hat{\theta}(r)((i(s+r)-u)+(i(s-r)-u))]
$$

Due to the similarity between Equation 7 and $8, f(r) \mathrm{s}$ behave like variances in different directions defined by $r$. Because of directionality, $f(r) \mathrm{s}$ can strengthen the orientation selectivity in texture feature detection, and have been proved to be more discriminatory than $\hat{\theta}(r) \mathrm{s}$ [2]. Hereby $f(r) \mathrm{s}$ and the variance $\hat{v}$ are employed as MRF features.

The performance of the GMRF approach depends very much on the neighbor set used. In Figure 1(a), up to $6^{\text {th }}$ order traditional neighbors are depicted. For example, a $2^{\text {nd }}$ order GMRF model centered on image cell $S$ would include those cells marked 1.x and 2.x. Traditional GMRF features are not effective for oriented texture classification, since there are limited directions defined. In order to obtain more orientation information of textures for any order model, we use a new neighbor set via the circular neighborhood system, which has been used by Kashyap and Khotanzad to achieve rotation-invariant features [6]. The circular neighbor sets for a $1^{\text {st }}$, $2^{\text {nd }}$, and $3^{\text {rd }}$ GMRF model with 16 orientations are shown in Figure $1(\mathrm{~b})$. An angular interval between two nearest neighbors in the same concentric circle is defined as $\theta=2 \pi / \tau$, where $\tau$ is the number of neighbors in one 
concentric circle. Note that the angular spacing of different concentric circle need not be the same, and the angular spacing of outer concentric circles can be smaller than that of inner circles. In this paper, we use the same angular spacing $22.5^{\circ}$ for all concentric circles. Finer quantization of orientation may be needed in some cases.

For simplicity, this paper refers to the GMRF model defined with traditional neighbors as a traditional GMRF (TGMRF) model while the GMRF model with circular neighbors as a circular GMRF (CGMRF) model. The CGMRF features can be computed using Equation 6-8, which is exactly the same as that of TGMRF features. The values of the neighbors that are not located on the image grid can be calculated by interpolation.

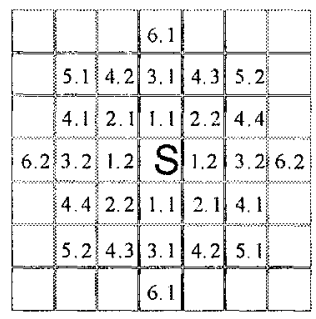

(a) traditional neighbor set

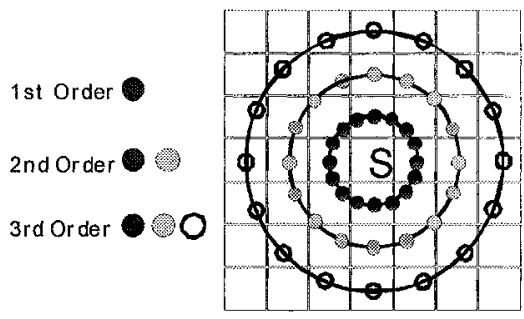

(b) circular neighbor set

Figure 1. Neighbor sets of GMRF models

\section{FEATURE FUSION WITH NEIGHBORHOOD- OSCILLATING TABU SEARCH (NOTS)}

The correlation relationship between MGF and CGMRF features is investigated. A low correlation coefficient (e.g., closer to zero), which suggests that the relationship between MGF and CGMRF features is weak or non-exist, shows the potential to produce an improved classification result by MGF/CGMRF feature fusion. In this section, the neighborhoodoscillating tabu search algorithm (NOTS) is proposed to solve the MGF/CGMRF feature fusion problem.

The strategy of feature fusion is first to combine various features and then perform feature selection to choose an optimal feature subset. A number of feature selection methods have been developed [7]. Tabu search has been proved to be a promising tool for feature selection in respect to the quality of obtained feature subset and computation efficiency, compared with classical algorithms, such as sequential forward selection (SFS) and sequential forward floating selection (SFFS) methods. The SFFS method yields better results than SFS, but it is still likely to trap into a local optimal solution. 
Tabu search differs from classical techniques, in that it allows the search to move away from the local minima in order to search more extensively in the feasible domain. There are some parameters which should be selected carefully, such as neighborhood size, tabu list's length and initial solution. An adaptive tabu tenure strategy has been proposed [8], and here we emphasize on the problem of the search neighborhood size. The classification process is based on the widely used multivariate Gaussian Bayes classifier and the Kappa coefficient $(\kappa)$ is used as the criterion of feature selection.

Traditional neighborhoods have constant sizes, for example, a 2neighborhood is generated by reversing one or two features' states [7]. When the neighborhood size is smaller, the computational efficiency is raised while the access to the neighborhood is limited. Alternatively, when the neighborhood size is bigger, the high-ration access to the neighborhood is maintained while computational cost is increased tremendously. Thus, we propose an oscillating neighborhood scheme by introducing the idea of the oscillating search algorithm [9]. The oscillating search is based on repeated of the current subset, which is achieved by alternating the down- and upswings. And the neighborhood size during tabu search is determined by the oscillation cycle depth. We call the tabu search method with the oscillating neighborhood scheme as neighborhood-oscillating tabu search (NOTS). Let $G$ denote the global best solution so far, $C$ the current best solution , $T L$ the tabu list, $o$ the oscillation cycle depth, $\Delta$ the used-specified limit of oscillation cycle depth, $t$ the number of none improvement at the current move and $T$ the maximum of consecutive rejections. Below is a detailed description of the NOTS algorithm.

Step 1. Initialization: generate the initial set $X$ of $d$ features by means of the SFS method. Let $G=X, C=X$, and $t=0$.

Step 2. Tabu moves: pick the best non-tabu neighbor in the dynamic neighborhoods using the oscillating search method. In the oscillating search, if the current feature set is better than $C$ but belongs to $T L$, we consider that the present search does not bring any improvement. The oscillating search algorithm stops if $C$ is better than $G$ or the value of $o$ exceeds $\Delta$.

Step 3. Output: If $C$ is better than $G$, let $G=C$ and $t=0$; or else let $t=t+1$. If $t \geq T$, the termination condition is satisfied, stop and output the global best solution $G$. Otherwise, let $T L=T L \cup C$ then go back to Step 2 . 


\section{EXPERIMENTAL RESULTS}

The experiment is set up on the following issues. First, we use NOTS to solve the MGF/CGMRF feature fusion problem, and compare the performance of NOTS with that of SFS and SFFS algorithms. Second, we test and compare the developing approaches with the traditional ones, namely TGF vs. MGF, and TGMRF vs. CGMRF, in a quantitative manner. It is expected that MGF and CGMRF features would respectively outperform TGF and TGMRF features. For both TGF and MGF, the filters with the two lowest frequencies are left out as they capture spatial variations that are too large to be considered texture. Third, the fused MGF/CGMF features achieved by NOTS are expected to achieve higher classification accuracy than either MGF or CGMRF features alone. All the extracted texture features should be normalized so that each measurement has zeromean and unit standard deviation before performance comparison.

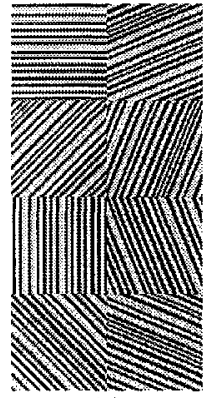

(a)

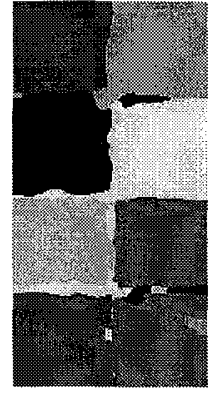

(b)

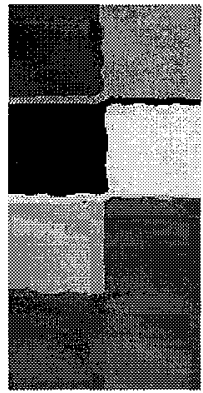

(c)

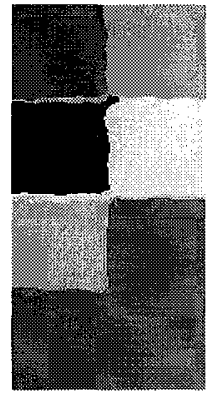

(d)

Figure 2. A comparison of classification results using SFS, SFFS, and NOTS methods to solve the MGF/CMGF feature fusion problem: (a) input image, (b) classification results using SFS, (c) classification results using SFFS, (d) classification results using NOTS.

Brodatz texture D49 (straw screening) is selected as the original texture. Figure 2(a) with size $128 \times 256$ is composed of 8 samples by rotating the texture with 8 angles $\left(0^{\circ}, 22.5^{\circ}, 45^{\circ}, 67.5^{\circ}, 90^{\circ}, 112.5^{\circ}, 135^{\circ}, 157.5^{\circ}\right)$ in the counterclockwise direction. First, Texture features are extracted from Figure 2(a) using MGF and the $2^{\text {nd }}$ order CGMRF model. For the $128 \times 256$ image, the MGF bank comprises 19 channels, resulting in a 19-dimensional MGF feature vector for each image pixel. And the $2^{\text {nd }}$ order CGMRF model corresponds to a 17-dimensional CGMRF feature vector for each point of the input image. Then SFS, SFFS, and NOTS methods are implemented to solve the MGF/CGMRF feature fusion problem, respectively. Figure 2 shows the graphical results for the three above algorithms while Table 1 lists the corresponding tabular results. The first column of Table 1 gives the dimensions of selected features (SD). The following columns give the Kappa 
coefficient $(k)$ and overall accuracy (OA) using that selected features. From Table 1, we can see that the NOTS method outperforms SFS and SFFS by yielding the Kappa coefficient of 0.9533 .

Table 1. Results using three feature selection algorithms for MGF/CGMRF feature fusion. SD-dimension of selected features, $k$ - Kappa coefficient, and OA - overall accuracy.

\begin{tabular}{llll}
\hline Feature Selection & SD & $k$ & OA \\
\hline SFS & 16 & 0.9389 & $94.64 \%$ \\
SFFS & 10 & 0.9348 & $94.29 \%$ \\
NOTS & 11 & 0.9533 & $95.92 \%$ \\
\hline
\end{tabular}

Table 2. Performance evaluation of different features. $D$ - dimension of features without selection, $S D$ - dimension of selected features using NOTS, $k$ - Kappa coefficient, OA overall accuracy, Max.J and Min. J - maximum and minimum Fisher criterion.

\begin{tabular}{lllllll}
\hline Feature type & $D$ & $S D$ & $k$ & OA & Max. J & Min. J \\
\hline TGF features & 12 & 6 & 0.8838 & $89.83 \%$ & 54167.24 & 744.58 \\
MGF features & 19 & 10 & 0.9040 & $91.60 \%$ & 200481.95 & 710.80 \\
TGMRF features & 5 & 3 & 0.7845 & $81.15 \%$ & 297.17 & 0.15 \\
CGMRF features & 17 & 8 & 0.9398 & $94.73 \%$ & 6919.36 & 379.77 \\
MGF \& CGMRF features & 36 & 11 & 0.9533 & $95.92 \%$ & 161218.65 & 1251.29 \\
\hline
\end{tabular}

For the sake of comparison, we also use TGF which comprises 12 channels and the $2^{\text {nd }}$ order TGMRF model, yielding a 12-dimensional TGF feature vector and a 5-dimensional TGMRF feature vector for each image pixel, respectively. Performance is assessed by the criterion of classification accuracy with the multivariate Gaussian Bayes classifier and NOTS. And the minimum and maximum Fisher criterions, which can express the separation ability between classes in the selected feature space, are recorded simultaneously. Table 2 shows that, as expected, the Kappa coefficient by MGF features is improved from 0.8838 to 0.9040 with respect to that by TGF features, and the Kappa coefficient by CGMRF features is increased from 0.7845 to 0.9398 with respect to that by TGMRF features.

Furthermore, the correlation relationship between the MGF and CGMRF features is investigated. The correlation coefficient between the two different types of features extracted from Figure 2(a) is 0.0473 . The low correlation coefficient indicates that CGMRF features are not well correlated with MGF features, and the combined features are expected to offer more texture information than the pure features. From Table 2, we can clearly see that the fused MGF/CGMRF features achieved by NOTS perform better than either MGF or CGMRF features alone. 


\section{CONCLUSIONS}

Based on traditional methods, this paper develops MGF and CGMRF techniques for oriented texture analysis. NOTS is proposed to solve the MGF/CGMRF feature fusion, and compared with SFS and SFFS methods. There are three important remarks made. First MGF and CGMRF features for oriented texture classification respectively outperform TGF and TGMRF features. Second NOTS, which would not trap into a local optimal solution, can find an optimal feature subset effectively, and its performance is better than that of SFS and SFFS methods. Finally according to classification accuracy, the MGF/CGMRF fused features achieved by NOTS perform better than either MGF or CGMRF features alone. The experimental results indicate that the proposed algorithm of MGF/CGMRF feature fusion with NOTS for oriented texture classification is stable and reliable and efficient to improve classification results.

\section{ACKNOWLEDGEMENTS}

This work was supported by the 973 Project of the People's Republic of China (Project Number 2003CB415205), and the National Natural Science Foundation of China (Project Number 40471088).

\section{REFERENCES}

[1] Jain A.K, et al. Unsupervised texture segmentation using Gabor filters. Pattern Recognition, 1991, 24(12): 1167 1186

[2] Cesmeli E, et al. Texture segmentation using Gaussian-Markov random fields and neural oscillator networks. IEEE Transactions on Neural Network, 2001, 12(2): 394 404

[3] Clausi D.A. Comparison and fusion of co-occurrence, Gabor, and MRF texture features for classification of SAR sea ice imagery. Atmosphere \& Oceans, 2001, 39(4): 183 194

[4] Clausi D.A, et al. Designing Gabor filters for optimal texture separability. Pattern Recognition, 2000, 33(11): 1835 1849

[5] Chellappa R, et al. Classification of textures using Gaussian Markov random fields. IEEE Transaction on Acoustics, Speech, and Signal Processing, 1985, 33(4): 959-963

[6] Kashyap R.L, et al. A model-based method for rotation invariant texture classification. IEEE Transactions on Pattern Analysis and Machine Intelligence, 1986, 8(4): 472 481

[7] Zhang H, et al. Feature selection using tabu search method. Pattern Recognition, 2002, 35(3): 701 711

[8] Korycinski D, et al. Adaptive feature selection for hyperspectral data analysis. SPIE The International Society for Optical Engineering, 2004, 5238: 213 225

[9] Somol P, et al. Oscillating search algorithms for feature selection. ICPR 2000: 406 2409 